\title{
BMJ Open Understanding the implementation (including women's use) of maternity waiting homes in low-income and middle-income countries: a realist synthesis protocol
}

\author{
Daphne N McRae (D) , ${ }^{1,2}$ Anayda Portela, ${ }^{3}$ Tamara Waldron, ${ }^{1}$ Nicole Bergen, ${ }^{4}$ \\ Nazeem Muhajarine ${ }^{1,2}$
}

To cite: McRae DN, Portela A, Waldron $\mathrm{T}$, et al. Understanding the implementation (including women's use) of maternity waiting homes in low-income and middleincome countries: a realist synthesis protocol. BMJ Open 2021;11:e039531. doi:10.1136/ bmjopen-2020-039531

- Prepublication history and additional material is published online only. To view please visit the journal online (http://dx.doi. org/10.1136/bmjopen-2020039531).

Received 21 April 2020

Revised 08 February 2021

Accepted 16 February 2021

Check for updates

(C) Author(s) (or their employer(s)) 2021. Re-use permitted under CC BY-NC. No commercial re-use. See rights and permissions. Published by BMJ.

For numbered affiliations see end of article.

Correspondence to Dr Nazeem Muhajarine; nazeem.muhajarine@usask.ca

\section{ABSTRACT}

Introduction Maternity waiting homes in low-income and middle-income countries provide accommodation near health facilities for pregnant women close to the time of birth to promote facility-based birth and birth with a skilled professional and to enable timely access to emergency obstetric services when needed. To date, no studies have provided a systematic, comprehensive synthesis explaining facilitators and barriers to successful maternity waiting home implementation and whether and how implementation strategies and recommendations vary by context. This synthesis will systematically consolidate the evidence, answering the question, 'How, why, for whom, and in what context are maternity waiting homes successfully implemented in low-income and middle-income countries?'. Methods and analysis Methods include standard steps for realist synthesis: determining the scope of the review, searching for evidence, appraising and extracting data, synthesising and analysing the data and developing recommendations for dissemination. Steps are iterative, repeating until theoretical saturation is achieved. Searching will be conducted in 13 electronic databases with results managed in Eppi-Reviewer V.4. There will be no language, study-type or document-type restrictions. Items documented prior to 1990 will be excluded. To ensure our initial and revised programme theories accurately reflect the experiences and knowledge of key stakeholders, most notably the beneficiaries, interviews will be conducted with maternity waiting home users/nonusers, healthcare staff, policymakers and programme designers. All data will be analysed using context-mechanism-outcome configurations, refined and synthesised to produce a final programme theory.

Ethics and dissemination Ethics approval for the project will be obtained from the Mozambican National Bioethical Commission, Jimma University College of Health Sciences Institutional Review Board and the University of Saskatchewan Bioethical Research Ethics Board. To ensure results of the evaluation are available for uptake by a wide range of stakeholders, dissemination will include peer-reviewed journal publication, a plain-language brief, and conference presentations to stakeholders' practice audiences.

\section{Strengths and limitations of this study}

- This study will provide a programme theory, based on a systematic consolidation of the global evidence, to explain successful (and unsuccessful) maternity waiting home implementation in low-income and middle-income countries.

- The integrated knowledge translation approach will enable coproduction of practical, relevant research that is applicable for women using maternity waiting homes, their families and communities, maternity waiting home staff, policymakers and programme designers.

- Realist methods will allow results to be tailored to various contexts to inform implementation strategies and recommendations.

- Due to resource constraints, interviews with maternity waiting home users/nonusers, healthcare providers, programme designers, implementers and policymakers will be conducted with a small number of participants, mainly from Mozambique and Ethiopia

PROSPERO registration number CRD42020173595.

\section{INTRODUCTION}

Background

Universal health coverage (UHC) is a primary focus of the UNs' Sustainable Development Goal 3.8, encompassing equitable provision of high-quality, accessible and affordable healthcare for all. ${ }^{1}$ Likewise, the Global Strategy for Women's and Children's Health focuses on the equitable promotion of women's and children's right to health, setting global maternal mortality targets at less than 70 per 100000 live births and newborn mortality at no more than 12 per 1000 live births by 2030 . In support of these goals, WHO released a 2018 guideline on essential labour and childbirth 
practices, to be provided by skilled health professionals, for safe birth. The guideline delineates minimum standards of practice that should be available to all pregnant women and their babies irrespective of socioeconomic setting. ${ }^{2}$ Despite progress towards these targets, in many low and middle-income countries (LMICs), significant barriers remain. Global goals will not be achieved until we address universal coverage of these packages, which are critical for safe birth.

The three delay model, proposed by Thaddeus and Maine (1994), describes the different type of delays women face in accessing care for obstetric complications. Since then, the model has been adapted and applied more broadly to describe delays in reaching services for routine childbirth and for obstetric complications. ${ }^{3}$ The first delay relates to the decision to access care. Decision-making is often dependent on cultural norms and health beliefs, whereby pregnant women are expected to defer decisionmaking to husbands/partners or family members. ${ }^{4}$ The second delay is caused by transportation issues, including lack of available transportation and infrastructure, long distances, poor road conditions and cost. The third delay is associated with a lack of timely and appropriate care on arrival at a health facility. ${ }^{3}$ Table 1 provides examples of the multiple explanatory factors that contribute to the first, second and third delay in the Ethiopian context ${ }^{4-6}$; studies from other LMICs report similar causes of delay for pregnant women seeking obstetric care. ${ }^{7-9}$

Maternity waiting homes (MWHs) have been used as a strategy in LMICs to address the first and/or second delay, with the aim of enabling timely access to facilitybased birth and birth with a skilled professional and to care for obstetric complications. ${ }^{10}$ While many different models exist, MWHs generally provide short-term accommodation for pregnant women (and sometimes a family member or chosen birth companion) during the late stages of pregnancy, within a short distance of a healthcare facility. ${ }^{11}$ Women staying at MWHs may access antenatal care from providers at affiliated health facilities and sometimes receive education and counselling on maternal and newborn healthcare issues (eg, symptoms of obstetric risk, sexual and reproductive health, and feeding and parenting practices) within the MWHs. ${ }^{12-14}$ Many MWHs also provide short-term, postnatal accommodation. MWHs have the potential to minimise inequity in perinatal service provision for women residing in rural and remote areas. Women from rural and remote areas have been shown to be less likely to engage with the healthcare system compared with those residing in urban areas. ${ }^{6}$

Studies have identified various determinants of MWH use and satisfaction, across a range of settings. These include cost of transportation to MWHs, food security within the MWHs, amount of time away from family or work,${ }^{10}{ }^{15}$ presence of antenatal complication, ${ }^{16}$ women's autonomy in decision-making ${ }^{6} 16$ and $\mathrm{MWH}$ conditions and service quality. ${ }^{6} 15$ 17-19 Despite the rich information available in individual studies, there have been no publications describing a comprehensive programme theory about how MWHs work as an intervention, specifically explaining why, for whom, under what circumstances and how MWHs are successfully (and unsuccessfully) implemented. To date, individual studies have provided various recommendations for improving MWH implementation, addressing a broad range of factors from structural design and governance of the homes, to provision of programming, services and material resources. ${ }^{15} 16$ 18-22 Nonetheless, we do not have an understanding of the applicability of these recommendations across diverging MWH interventions, in differing contexts or for various populations, nor has there been an evaluation of the theories underpinning MWH implementation strategies. Using realist synthesis, this study will uniquely contribute to the literature and to practice by providing an evidencebased programme theory of MWH implementation, including an explanation of facilitators and barriers to women's use, which can be tailored to various contexts.

\section{Realist philosophy and methodology}

The factors determining the successful implementation of maternal healthcare services in LMICs in general, and MWHs specifically, are multifactorial, involving individual, family, community, health system and country-level and global-level influences, shaped by cultural, ethnic and religious beliefs as well as socioeconomic, political and geographic elements. In order to tease apart what works,

Table 1 Factors contributing to the three delay model for pregnant women in Ethiopia

\begin{tabular}{ll}
\hline Delay & Contextual factors \\
\hline $\begin{array}{l}\text { 1.Delay in healthcare } \\
\text { seeking behaviour }\end{array}$ & Religious influences \\
& Familial responsibilities \\
& Husband and mother-in-law preferences \\
& Negative previous experience at health \\
& facility \\
& Positive previous experience of home \\
& birth \\
& Lack of awareness of pregnancy-related \\
& problems \\
& Unable to afford services \\
& Self-efficacy \\
2.Delay in arriving at & Lack of transportation \\
Fa health facility & Far distance to travel \\
& Road conditions impassable \\
& Financial constraints \\
& Delayed referral \\
& Labour started at night \\
& Visiting several accessible but non- \\
functioning health facilities
\end{tabular}


for whom, in what circumstances, how and why or why not, there is a need to evaluate the full body of information, using methods that account for complexity. ${ }^{23}$ Realist philosophy bridges constructivism, which posits that the world is a socially produced reality, with meaning formed through human interpretations of social interactions, and positivism, which holds that truth is derived from observable, measurable factors interpreted through reason and logic. ${ }^{24}$ Realist philosophy acknowledges that observable truth exists in the world, but that the meaning of truth varies by individual perception, through the influence of cultural and social impacts. ${ }^{25}$ Two main methods of realist inquiry are realist evaluation and realist synthesis. Whereas realist evaluation uses primary data, realist synthesis (the focus of this protocol) uses systematic review and analysis of mainly secondary sources to identify, test and refine programme theory. ${ }^{26}$ Both methods aim to provide a theoretical explanation of how interventions and programmes are anticipated to meet their objectives via the role of observable and unobservable factors. ${ }^{23} 27$

Realist inquiry describes explanatory mechanisms, such as motivation or distrust, in specific contexts that generate intended or unintended outcomes. ${ }^{23}{ }^{28}$ From a realist perspective, an outcome is the product of the interaction between context (ie, an enabling environment) and underlying mechanisms-specifically the resources provided by the programme or intervention and the participants' response to them. ${ }^{29}$ Mechanisms describe the interaction between the pre-existing context and resulting outcomes. ${ }^{25}$ Realist evaluation and synthesis are built on the generation and testing of multiple evidencebased hypotheses, constructed as context-mechanismoutcome configurations (CMOCs). ${ }^{23}$ The configurations are supported by formal theories (grand theories already tested and described in the literature) to understand the intrinsic generative causal processes. In realist evaluation or synthesis, CMOCs amalgamate to create a programme theory, an explanation of the underlying reasons why, how and for whom the programme works or does not work. Realist reasoning asserts that specific pre-existing contexts influence individuals' responses to programme resources causing them to make choices in expected or predictable patterns. ${ }^{30}$ These patterns, referred to as demiregularities, observed within and between CMOCs can clarify and provide evidence in support of hypothesised programme theories, in turn illuminating how and why a programme is or is not successful in meeting its objectives. $^{27}$

Realist syntheses are conducted retrospectively, drawing from the body of evidence on a topic, with the purpose of developing explanatory programme theory at the middlerange level of abstraction. The resulting programme theory is a series of testable hypotheses that can be evaluated in different contexts to determine the validity and usefulness of the theory in explaining programme effects. ${ }^{30}$ It is programme theory, then, that is continually tested and stands to be generalised, rather than programme activities or outcomes. All types of secondary data (such as quantitative, qualitative and mixed methods research, case studies, editorials, reports and social media posts) are eligible to inform and revise a developing programme theory. ${ }^{30}$ Realist synthesis has practical implications as the resulting programme theory can direct how a programme could be structured, modified or targeted to a specific population or context to maximise success, when it is applied in new settings or contexts. In addition, fully developed programme theories can be used for designing and evaluating similar types of programmes.

\section{Rationale for conducting an MWH realist synthesis}

The type of programmes especially well suited to realist synthesis includes those that are complex and explicitly or implicitly assume that a programme will instigate some type of action among participants (eg, change in behaviour or reasoning) triggering intended outcomes. ${ }^{23}$ MWH interventions meet both the criteria: (1) they are complex, dependent on interwoven supply and demand issues and shaped by the multiple actors and social systems involved and (2) it is assumed that the provision of MWHs will cause more women to migrate towards health facilities near the end of pregnancy than if MWHs were not available. ${ }^{31}$ Moreover, realist synthesis is an appropriate method because it permits investigation of the entire MWH implementation chain, from the supply-side (eg, health system resources and support for MWHs) through to factors influencing the demand-side, such as users' knowledge of MWH availability, home and family dynamics, sociocultural and accessibility considerations. ${ }^{23} 32$ The breadth and depth of investigation allow for comprehensive, evidence-informed recommendations. Practically, identifying a programme theory rather than merely the activities associated with MWHs will advance the current and future applications of this intervention and produce the results that it promises in maternal and newborn health.

\section{Research objective}

In this article, we outline the protocol for a realist synthesis to better understand successful (and unsuccessful) MWH implementation in LMICs. The realist synthesis will answer the question, 'How, why, for whom and in what context are MWHs successfully implemented in LMICs?'. This question will be answered by examining the literature and consulting with key stakeholders to decipher how MWH contexts interact with causal mechanisms to encourage or inhibit MWH implementation. Empirically tested CMOCs that explain MWH implementation will be compared against our initial programme theory (IPT) to refine it into a final programme theory. By default, conditions leading to less successful MWH implementation will become apparent from this study. Less successful MWH implementation will be characterised by contexts that fail to trigger the causal mechanisms associated with highly successful implementation. 


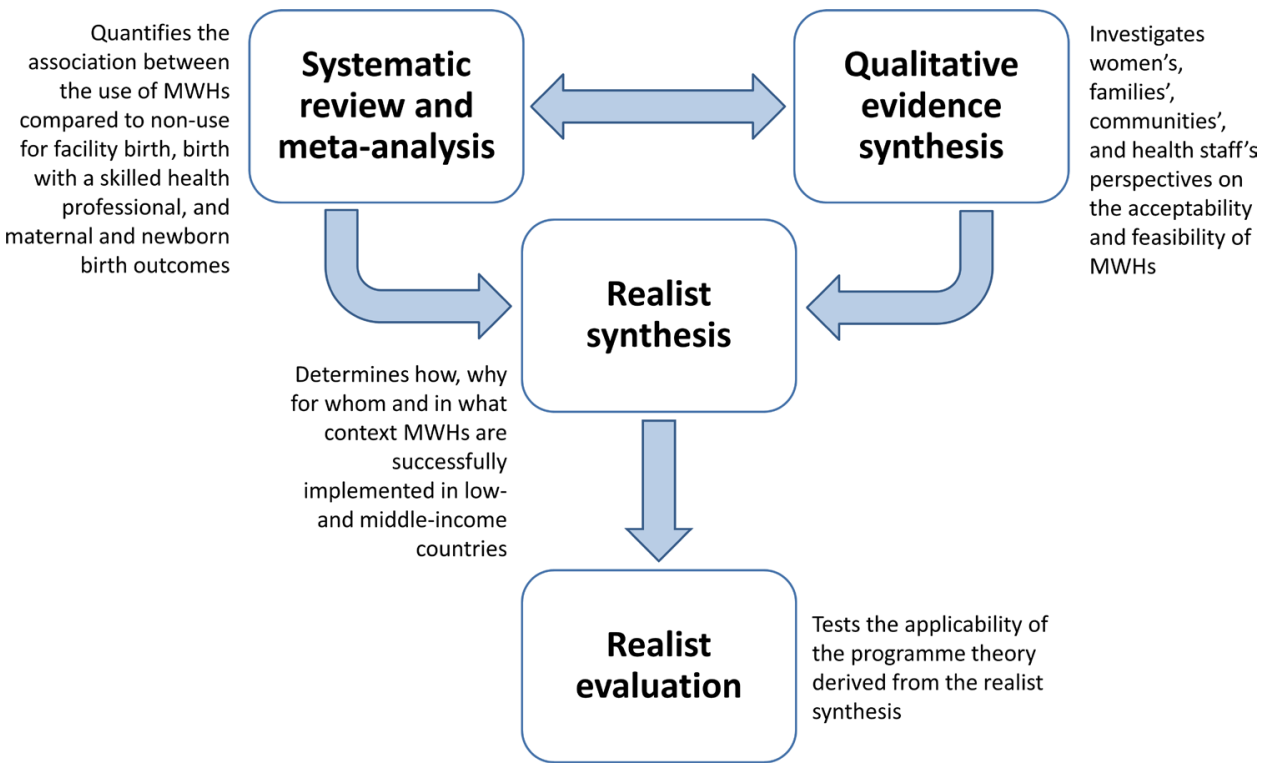

Figure 1 Consolidating the global MWH evidence via three reviews and a realist evaluation. MWH, maternity waiting home.

'Successful implementation', our primary outcome, will be explored and defined iteratively, considering multiple aspects of implementation and various stakeholder perspectives. For example, primary indicators of 'successful implementation' include women's and families' satisfaction, awareness and use of MWHs. Other important aspects may include community, healthcare provider and policymaker support for MWHs, with support demonstrated through a sense of community ownership, provider referral and fiscal commitment. Successful MWH implementation may also be demonstrated by sustainability of services over time, replicability in neighbouring districts, fidelity to $\mathrm{MWH}$ programmes and their intended use and integration of MWHs into a health service systems. ${ }^{33}$ As 'success' is a broadly definable concept, we anticipate identifying other indicators of successful implementation throughout the evaluation process.

This study is part of a review package, including three comprehensive reviews and a realist evaluation (see figure 1) designed to understand the effectiveness, acceptability, feasibility and successful implementation of MWHs. The realist synthesis will build on the results of a systematic review, be informed by a qualitative evidence synthesis and, in turn, its programme theory is evaluated by a country-specific realist evaluation. The systematic review and meta-analysis, currently being conducted, will quantify the association between the use of MWHs and facility births, birth with a skilled health professional and maternal and newborn health outcomes for users compared with nonusers. These results allow estimates of the clinical and policy impact of MWHs. The qualitative evidence synthesis will investigate women's, families', communities' and health staff's perspectives on the acceptability and feasibility of MWHs. Findings from this study will explain what stakeholders perceive to be key issues and how perceptions differ by population group. These results will be useful for considering various groups' needs in design, implementation and refinement of MWHs.

Results of all three studies will be used by the Mozambique-Canada Maternal Health Project, ${ }^{34}$ to guide $\mathrm{MWH}$ programme implementation for three newly constructed MWHs in Inhambane, Mozambique. Additionally, a realist evaluation will test the applicability of the programme theory derived from this realist synthesis against women's experiences in the new MWHs.

\section{METHODS}

This study uses an integrated knowledge translation (IKT) approach, impacting the design, analysis and dissemination of results. IKT is a collaborative research method in which researchers and knowledge users coproduce practical, applicable research for the knowledge users' communities. ${ }^{35}$ Researchers supply methodological and content expertise while knowledge users provide experiential and context expertise and are positioned to implement research recommendations. This approach can produce results that more accurately reflect reality and are of greater pertinence to users, build research capacity and encourage policy and practice change. ${ }^{35}$ The current realist synthesis team includes five researchers. Two members have previous experience conducting realist research $(\mathrm{NM}, \mathrm{TW})$, four have expertise in $\mathrm{MWH}$ research and evidence synthesis (DNM, NM, NB, AGP) and all have been trained in qualitative and/or quantitative methods. Key stakeholders will be interviewed to confirm, refute and revise the emerging programme theory, thereby ensuring those with lived experience and expertise contribute to the analysis. Informal consultation with other experts will be on-going throughout the theory development process and will facilitate dissemination and uptake of results among stakeholder practice audiences. 


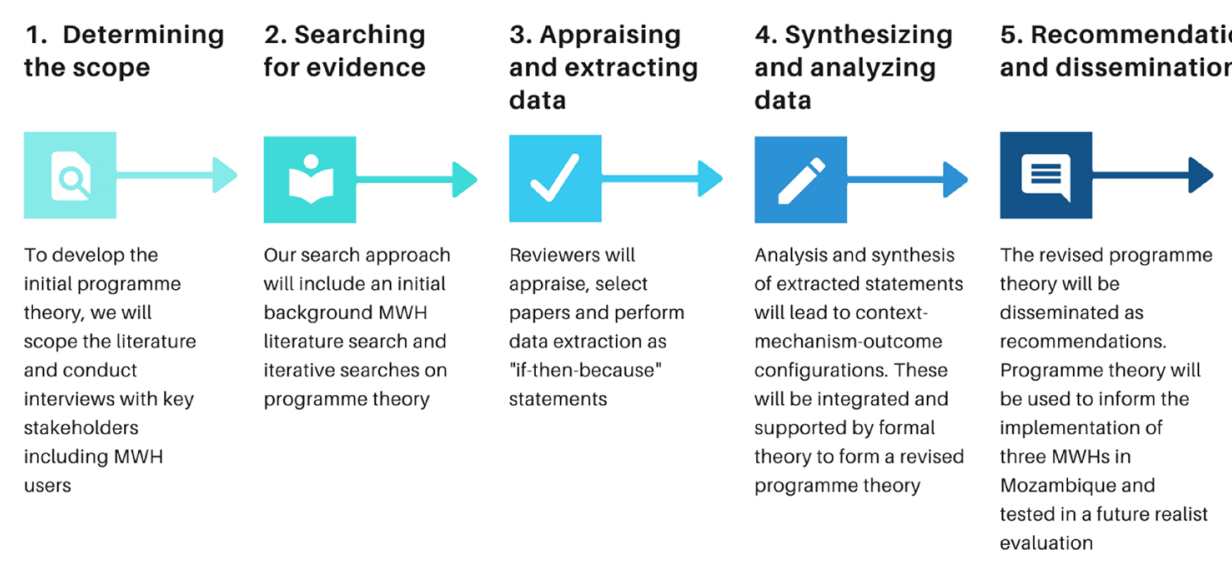

Figure 2 Methods for iteratively developing initial and final programme theory in realist synthesis. ${ }^{23} \mathrm{MWH}$, maternity waiting home.

Our study will be conducted between September 2020 and May 2021. It is designed and will be reported according to 'Realist and Meta-Review Evidence Synthesis: Evolving Standards' (RAMSES), publication standards for realist syntheses. ${ }^{36}$ The synthesis protocol was registered with the International Prospective Register of Systematic Reviews (PROSPERO). Our study design follows standard steps for realist synthesis, previously outlined by Pawson $e t$ $a l^{23}$ These include determining the scope of the review, searching for evidence, appraising and extracting data, synthesising and analysing data and developing recommendations and disseminating results (figure 2).

\section{Scoping the evidence}

Our first step will be to scope the literature to gather information for developing an IPT. ${ }^{23}$ We will glean general information pertaining to MWH service implementation (eg, differing definitions, characteristics and strategies of MWHs and common barriers/facilitators to uptake) to gain an overview of the key issues. Simultaneously, we will note any theories, assumptions and rationales explaining facilitators and barriers to MWH implementation within the literature. Theories specific to MWHs or pertaining to maternity care service interventions (eg, hostels for women awaiting fistula surgery) and any substantive social science theories (eg, the Health Belief Model) will be added to a list for future investigation.

\section{Search process}

Our search approach will be guided by a six-component framework outlined by Booth and colleagues for realist searching. ${ }^{37}$ Within this framework, the first search is conducted to formulate the research question, the second search is to supply background information. We have completed these searches as a part of a grand search to locate all MWH articles in 13 databases for the three MWH reviews. Our research question has been formulated as per RAMSES reporting standards. Three more iterative searches will be conducted to provide information on programme theory and empirical evidence and to refine the programme theory. The final step will be ensuring complete documentation of the search process. ${ }^{37}$
In the search to inform our research question and provide background information, we sought to identify all MWH articles included in the peer-reviewed and grey literature found in 13 electronic databases (AMED, CINAHL, Cochrane Library-Pregnancy and Childbirth, EMBASE, Global Health, Google Scholar, LILACS, MASCOT/WOTRO, OVID Medline, ProQuest Dissertations and Theses Global, PsychInfo, Pubmed and Web of Science). As MWHs are prevalent in Latin American and African countries, during our original search, we explored Spanish, Portuguese and French phrases for 'maternity waiting home' in Google Scholar. Finding Spanish and Portuguese documents, we added Spanish and Portuguese search terms to our search strategy. We searched titles and abstracts, adapting the following terms to meet each database's search requirements: (( (wait* OR await*) and (facilit* OR home* OR hut* OR shelter* OR area* OR ward*)) adj5 (antenatal* OR prenatal* OR pregnan* OR mother* OR matern* OR birth* OR intrapartum OR labour OR labor OR childbirth OR childbearing OR "child bearing" OR deliver*) OR ("casa* materna*" OR "hogar* materno*" OR "casa de parto" OR "casa da gestante" OR "casa de gestante" OR "bebê e puérpera" OR "centro de parto humanizado" OR "centro de parto normal")) (see the full search strategy in online supplemental appendix A). The following additional eligibility criteria were applied: (1) items had to be dated no early than 1990, (2) the setting was an LMIC, (3) items had to include a discussion of MWHs, defined as any structure located within or near a health facility, that provides (at least) antenatal accommodation for pregnant women and exists to enable women to access (a) skilled health professional(s) during childbirth or earlier if complications arise. There was no language, study-type or documenttype restrictions. Retrieved studies were imported into Eppi-Reviewer V.4 for data management. ${ }^{38}$

For our second search, on programme theory, we will conduct a topic search using our Eppi-Reviewer V.4 MWH database and the following terms (model* OR theor* OR concept* OR framework*). ${ }^{39}$ Searching using the topic function will enable us to retrieve articles that have 
a theoretical component but do not explicitly state the theory in the title or abstract. ${ }^{39}$ Simultaneously, we will conduct our third search, a title and abstract search in the above 13 databases using the following terms: (antenatal* or prenatal* OR pregnan* OR mother* OR matern* OR birth* OR intrapartum OR labour OR labor ORchildbirth OR childbearing OR "child bearing" OR deliver*) AND (( (health or medical) and (service* or care)) or healthcare or health-care) AND (model* OR theor* OR concept* OR framework*) AND (implement* OR intervention* OR program* OR initiative* OR strategy* OR approach* OR action* OR project* OR activit*) (see the full search strategy in online supplemental appendix A). This third search will allow us to locate theories explaining implementation of maternal health services in general, in addition to theories explaining implementation of MWHs. Depending on the number of items located, we may limit the search to theories explaining implementation of maternal health services in LMICs.

After all searches and screening are complete, we will compile a grand list of theories. On expert consultation, other theories may be added to the list and those most relevant will be selected for further investigation, to determine whether they have the potential to contribute to the IPT. For those theories selected, key citations will be sought and citation searches will be conducted. ${ }^{39}$ If warranted, subsequent searches will be conducted to retrieve information on specific aspects of individual theories. ${ }^{37}$ The search strategy may be modified and expanded to incorporate other relevant terms and concepts as they become apparent. Authors may be contacted to request further information about their work and to discuss their knowledge of other relevant sources.

In addition to searching the literature, information will be derived from interviews with key stakeholders (eg, MWH users/nonusers and staff, practitioners from facilities associated with MWHs and healthcare policymakers) in Mozambique and Ethiopia as well as MWH programme designers and researchers from elsewhere. Interviewees will be asked to vet our evolving IPT against their lived experience with MWHs, to confirm or refute our hypothesised CMOCs. ${ }^{37}$ In later stages, interviews will be conducted to discuss multiple and competing theories with stakeholders and verify and/or refine our final programme theory.

To recruit participants, we will capitalise on contacts made through existing maternal health projects in Mozambique (led by NM) and Ethiopia (involving team member NB). Other global health researchers with MWH expertise will be identified by team members or via MWH publications and snowballing methods. Our contact information and a recruitment email will be provided to our MWH contacts and we will request that they forward this information to any potential participants. Interviews will be 1 hour long, conducted on an online video conferencing platform or by phone. Interviews will be conducted by an interviewer and a notetaker, both who are familiar with the programme theory and able to formulate impromptu questions specific to the causal mechanisms that participants name as a part of their response to planned questions. Participants will have the option of interviewing in English or in the language of their choice with a translator. Our initial interview guide will be informed by Westhorp's and Manzano's 'starter set' of realist interview questions (see online supplemental appendix B).$^{40}$ Interviews will be recorded (with permission), transcribed and, if necessary, translated into English. All interview transcripts will be imported into NVivo $^{41}$ for analysis, with interviewers' field notes and reflections appended.

\section{Selection, appraisal and data extraction}

All retrieved title and abstract citations will be uploaded in Eppi-Reviewer V.4. ${ }^{38}$ After deduplication, items will be screened by title and abstract according to their relevance to the synthesis' objectives. ${ }^{23}$ Selected items must have the potential to inform theory and contain adequate descriptive information to contribute towards the development of CMOCs. We anticipate that inclusion and exclusion criteria will evolve as we become more familiar with the material and discuss the findings as a team. ${ }^{36}$

Two team members will review items. They will export those selected for full-text review to NVivo, ${ }^{41}$ taking notes and creating annotations on the theory and hypothesised CMO relationships reported in each source. We will identify the relevant outcome (aspects of successful MWH implementation) in each article and create 'if ... then ... because' statements to describe what activates outcomes, how, and in which contexts. ${ }^{36}$ A data extraction template will be used to capture standard bibliographic information, including the study's country of origin as well as descriptions of the focus of the research/document, sample population, MWH intervention, contextmechanism-outcome elements, study's relevance to the programme theory, and whether it is an empirical or theoretical article. ${ }^{42}$

Initially, two reviewers will work together selecting and extracting data to ensure consistent selection and note taking. Once reliability is apparent, team members will independently review $10 \%$ of the data for selection and data extraction. These items will then be re-examined by a third reviewer to measure selection inter-rater reliability ${ }^{43}$ using the Kappa statistic. If Kappa is greater than 80, the remaining items will be divided between reviewers. If the Kappa statistics is less than 80 , we will conduct double screening and/or extraction, with diverging classifications discussed between reviewers to reach consensus or, when necessary, deferring final decisions to the full review team. Excluded items and the rationale for exclusion will be documented as well as information on discrepancies in reviewers' decisions.

After initial selection, items will be reviewed for rigourexamined to evaluate whether credible and trustworthy methods have been used for inquiry. ${ }^{36}$ Not all aspects or even the main components of an article need to be relevant or meet the criteria for rigour or for the item to be 
included in the review; only the pertinent sections of the source will be evaluated for inclusion. ${ }^{23}$ Two reviewers will assess the rigour of all selected items. A $10 \%$ random sample will be classified by a third, blinded reviewer to evaluate consistency in quality assessment.

\section{Analysis and synthesis processes}

The purpose of this stage is to revise the IPT and form a refined programme theory. To achieve this, each part of the IPT will be analysed, comparing its theoretical claims against the collected data. ${ }^{44}$ Data will be collated by grouping 'if ... then ... because' statements according to MWH user characteristics (such as distance between residence and the nearest health facility, material resources provided at the MWH and sources of financial support for the $\mathrm{MWH}$ ). We will then analyse the data to determine demiregularities between contexts and outcomes. This will facilitate the identification of mechanisms that contribute to predictable patterns of CMOCs affecting programme functioning. ${ }^{28}$ Demiregularities will be used to sort CMOCs into thematic groups. If a CMOC fits into more than one thematic group, it will be added to all relevant groups. The thematic groups will then be analysed to assess the need for further classification (eg, by demand vs supply factors). Informed by the literature and developed through team discussions, we will seek to explain the patterns observed, theorising and drawing on existing middle-range theory where applicable. ${ }^{36}$

Multiple and competing theories will be discussed among our research team and in consultation with key stakeholders and experts to confirm, refine or refute the validity of our hypothesised CMOCs. ${ }^{44}$ Refined theories will be consolidated into a final programme theory. ${ }^{44} \mathrm{~A}$ narrative synthesis will be reported and supported by explanatory diagrams and tables.

The final deliverable will be a middle-range level programme theory that refines our understanding of how MWHs are successfully implemented, considering contextual factors. The programme theory will be founded on the evidence-based CMOC hypotheses, described in a manner that can be empirically tested. The results of the synthesis will include practical recommendations, with contextual caveats, applicable to MWH policy and practice in LMICs. $^{44}$

\section{Recommendations and dissemination}

Among our current team members, recommendations from this work will have particular relevance as we are planning and/or engaged in the structural and programme design of three MWHs in Mozambique through the Mozambique-Canada Maternal Health Project (NM, DNM), an MWH randomised cluster intervention trial in Jimma Zone, Ethiopia (NB) and an update of global recommendations for MWH use (AGP). In addition, programme theory resulting from the realist synthesis will be tested in a realist evaluation, conducted with key stakeholders involved with the three new MWHs in Inhambane, Mozambique. Results of the synthesis will be disseminated through peer-reviewed journal publications, a plain-language brief and conference presentations to stakeholders' practice audiences.

\section{Patient and public involvement}

Women who are eligible to use MWHs, and their families and communities, will be involved in the research in several capacities. The primary outcome, successful implementation, will be defined in part by stakeholder perspectives and experiences. The interviews with key stakeholders, initiated early in the research process, will ensure that beneficiary priorities are considered in the evolving search and analysis phases. In addition, women in Mozambique and Ethiopia will be involved in data collection including participant recruitment, conducting interviews, transcription and translation. Interviewees will also be asked to provide input into dissemination planning (content, medium, audience) and will benefit from presentations made within their local communities.

\section{ETHICS}

Ethics approval is generally not required for systematic reviews/syntheses using secondary data. However, because we will be conducting interviews among key stakeholders in Mozambique and Ethiopia, we will obtain ethics approval for the project from the Mozambican National Bioethical Commission, Jimma University College of Health Sciences Institutional Review Board and the University of Saskatchewan Bioethical Research Ethics Board.

\section{CONCLUSION}

Realist synthesis probes beyond assessments of effectiveness, in search of understanding why and how, for whom and in what context programmes or interventions, do or do not lead to intended outcomes. ${ }^{28}$ Realist methods allow researchers' to infer evidence-informed theories and test their validity. ${ }^{28}$ Using this approach will broaden our understanding of the means to improving MWH impact, policy and programming, including evidence-based guidance for the design and implementation of new MWHs. The result of this synthesis will be a programme theory that depicts how MWHs do or do not work, in which situations, and why.

\section{Author affiliations}

${ }^{1}$ Department of Community Health and Epidemiology, University of Saskatchewan College of Medicine, Saskatoon, Saskatchewan, Canada

${ }^{2}$ Saskatchewan Population Health and Evaluation Research Unit, Saskatoon,

Saskatchewan, Canada

${ }^{3}$ Department of Maternal, Newborn, Child and Adolescent Health and Ageing, World Health Organization, Geneva, Switzerland

${ }^{4}$ Interdisciplinary School of Health Sciences, University of Ottawa, Ottawa, Ontario, Canada

Twitter Anayda Portela @anagportela and Tamara Waldron @tamara_waldron1 Contributors NM conceptualised and conceived of the study and is the principal investigator of the Mozambique-Canada Maternal Health Project, through which 
the funds for this study have been procured. DNM, TW, NB and AGP contributed to the conceptualisation of the study. DNM drafted the original manuscript, all authors provided substantive content revision. All authors read and approved the final manuscript.

Funding This work is supported by funding from Global Affairs Canada (project number D-002085) for the Mozambique-Canada Maternal Health Project, administered by the University of Saskatchewan. The funder was not involved in any aspect of the study protocol.

Competing interests None declared.

Patient consent for publication Not required

Provenance and peer review Not commissioned; externally peer reviewed.

Supplemental material This content has been supplied by the author(s). It has not been vetted by BMJ Publishing Group Limited (BMJ) and may not have been peer-reviewed. Any opinions or recommendations discussed are solely those of the author(s) and are not endorsed by BMJ. BMJ disclaims all liability and responsibility arising from any reliance placed on the content. Where the content includes any translated material, BMJ does not warrant the accuracy and reliability of the translations (including but not limited to local regulations, clinical guidelines, terminology, drug names and drug dosages), and is not responsible for any error and/or omissions arising from translation and adaptation or otherwise.

Open access This is an open access article distributed in accordance with the Creative Commons Attribution Non Commercial (CC BY-NC 4.0) license, which permits others to distribute, remix, adapt, build upon this work non-commercially, and license their derivative works on different terms, provided the original work is properly cited, appropriate credit is given, any changes made indicated, and the use is non-commercial. See: http://creativecommons.org/licenses/by-nc/4.0/.

\section{ORCID iD}

Daphne N McRae http://orcid.org/0000-0001-8113-9028

\section{REFERENCES}

1 World Health Organization. What is universal health coverage? Geneva: WHO, 2014. Available: www.who.int/features/qa/universal health_coverage/en/ [Accessed cited 2019 July 25]

2 World Health Organization. WHO recommendations: intrapartum care for a positive childbirth experience. Geneva: WHO, 2018. Available: apps.who.int/iris/bitstream/handle/10665/260178/9789241550215eng.pdf [Accessed cited 2019 July 25].

3 Thaddeus S, Maine D. Too far to walk: maternal mortality in context. Soc Sci Med 1994;38:1091-110.

4 Berhan Y, Berhan A. Commentary: Reasons for persistently high maternal and perinatal mortalities in Ethiopia: Part III-Perspective of the "three delays" model. Ethiop J Health Sci 2014;24:137-48.

5 Bergen N, Labonté R, Asfaw S. Equity in maternal, newborn and child health: expanding health services onto rural Ethiopia. In: Cochrane L, ed. Ethiopia: social and political issues. Hauppauge, NY: Nova Publishers, 2019: 91-124.

6 Bergen N, Abebe L, Asfaw S, et al. Maternity waiting areas - serving all women? Barriers and enablers of an equity-oriented maternal health intervention in Jimma Zone, Ethiopia. Glob Public Health 2019;14:1509-23.

7 Ngoma-Hazemba A, Hamomba L, Silumbwe A, et al. Community perspectives of a 3-Delays model intervention: a qualitative evaluation of Saving Mothers, Giving Life in Zambia. Glob Health Sci Pract 2019;7:S139-50.

8 Mgawadere F, Unkels R, Kazembe A, et al. Factors associated with maternal mortality in Malawi: application of the three delays model. BMC Pregnancy Childbirth 2017;17:219.

9 Sk MIK, Paswan B, Anand A, et al. Praying until death: revisiting three delays model to contextualize the socio-cultural factors associated with maternal deaths in a region with high prevalence of eclampsia in India. BMC Pregnancy Childbirth 2019;19:314.

10 Lori JR, Wadsworth AC, Munro ML, et al. Promoting access: the use of maternity waiting homes to achieve safe motherhood. Midwifery 2013;29:1095-102.

11 World Health Organization Dept. of Reproductive Health and Research. Maternity waiting homes: a review of experiences. Geneva: WHO, 1996. Available: https://apps.who.int/iris/bitstream/ handle/10665/63432/WHO_RHT_MSM_96.21.pdf?sequence=1 [Accessed cited 2019 June 30].

12 Eckermann E, Deodato G. Maternity waiting homes in Southern Lao PDR: the unique 'silk home'. J Obstet Gynaecol Res 2008;34:767-75.
13 García Prado A, Cortez R. Maternity waiting homes and institutional birth in Nicaragua: policy options and strategic implications. Int $J$ Health Plann Manage 2012;27:150-66.

14 Gorry C. Cuban maternity homes: a model to address at-risk pregnancy. MEDICC Rev 2011;13:12-15.

15 Vermeiden T, Schiffer R, Langhorst J, et al. Facilitators for maternity waiting home utilisation at Attat Hospital: a mixed-methods study based on 45 years of experience. Trop Med Int Health 2018;23:1332-41.

16 Sialubanje C, Massar K, Hamer DH, et al. Personal and environmental factors associated with the utilisation of maternity waiting homes in rural Zambia. BMC Pregnancy Childbirth 2017;17:136

17 Sialubanje C, Massar K, van der Pijl MSG, et al. Improving access to skilled facility-based delivery services: women's beliefs on facilitators and barriers to the utilisation of maternity waiting homes in rural Zambia. Reprod Health 2015;12:61.

18 Mclntosh N, Gruits P, Oppel E, et al. Built spaces and features associated with user satisfaction in maternity waiting homes in Malawi. Midwifery 2018;62:96-103.

19 Ruiz MJ, van Dijk MG, Berdichevsky K, et al. Barriers to the use of maternity waiting homes in Indigenous regions of Guatemala: a study of users' and community members' perceptions. Cult Health Sex 2013;15:205-18.

20 Scott NA, Vian T, Kaiser JL, et al. Listening to the community: using formative research to strengthen maternity waiting homes in Zambia. PLoS One 2018;13:e0194535.

21 Lori JR, Munro-Kramer ML, Shifman J, et al. Patient satisfaction with maternity waiting homes in Liberia: a case study during the Ebola outbreak. J Midwifery Womens Health 2017;62:163-71.

22 Mramba L, Nassir FA, Ondieki C, et al. Reasons for low utilization of a maternity waiting home in rural Kenya. Int $J$ Gynaecol Obstet 2010;108:152-3.

23 Pawson R, Greenhalgh T, Harvey G, et al. Realist review--a new method of systematic review designed for complex policy interventions. J Health Serv Res Policy 2005;10 Suppl 1:21-34.

24 Greenhalgh T, Wong G, Westhorp G, et al. Protocol--realist and meta-narrative evidence synthesis: evolving standards (RAMESES). BMC Med Res Methodol 2011;11:115.

25 Pawson R, Tilley N. Realistic evaluation. Thousand Oaks, California: SAGE, 1997.

26 et alGreenhalgh T, Pawson R, Wong G. Realist evaluation, realist synthesis, realist research-what's in a name? The RAMSES II Project. National Insitute for Health Research, 2017. Available: http://www. ramesesproject.org/media/RAMESES_II_RE_RS_RR_whats_in_a_ name.pdf [Accessed cited 2020 Jan 29].

27 Dieleman M, Wong G, Marchal B. An introduction to realist research workshop. Bejing: second global symposium on health systems research, 2012. Available: https://www.abdn.ac.uk/femhealth/ documents/Realist_methods_workshop.pdf

28 Jagosh J, Pluye P, Wong G, et al. Critical reflections on realist review: insights from customizing the methodology to the needs of participatory research assessment. Res Synth Methods 2014:5:131-41.

29 Dalkin SM, Greenhalgh J, Jones D, et al. What's in a mechanism? development of a key concept in realist evaluation. Implement Sci 2015;10:49.

30 Rycroft-Malone J, McCormack B, Hutchinson AM, et al. Realist synthesis: illustrating the method for implementation research. Implement Sci 2012;7:33.

31 World Health Organization. WHO recommendation on establishment of maternity waiting homes (MWHs). WHO reproductive health library. Geneva: WHO, 2015. Available: extranet.who.int/rhl/topics/ improving-health-system-performance/who-recommendationestablishment-maternity-waiting-homes-mwhs [Accessed cited 2019 July 25].

32 Kurji J, Gebretsadik LA, Wordofa MA, et al. Factors associated with maternity waiting home use among women in Jimma zone, Ethiopia: a multilevel cross-sectional analysis. BMJ Open 2019;9:e028210.

33 Brennan N, Bryce M, Pearson M, et al. Understanding how appraisa of doctors produces its effects: a realist review protocol. BMJ Open 2014;4:e005466.

34 Muhajarine N. New research partnership makes childbirth safer in Mozambique. The Conversation, 2018. Available: https:// theconversation.com/new-research-partnership-makes-childbirthsafer-in-mozambique-93771 [Accessed cited 2019 April 14]

35 Kothari A, McCutcheon C, Graham ID. Defining integrated knowledge translation and moving forward: a response to recent commentaries. Int J Health Policy Manag 2017;6:299-300.

36 Wong G, Greenhalgh T, Westhorp G, et al. RAMESES publication standards: realist syntheses. BMC Med 2013;11:21. 
37 Booth A, Briscoe S, Wright JM. The "realist search": A systematic scoping review of current practice and reporting. Res Synth Methods 2020;11:14-35.

38 Thomas J, Brunton J, Graziosi S. EPPI-Reviewer 4: software for research synthesis. EPPI-Centre software. London: Social Science Research Unit, UCL Institute of Education, 2010. (cited 2019 Nov 15).

39 Booth A, Carroll C. Systematic searching for theory to inform systematic reviews: is it feasible? is it desirable? Health Info Libr J 2015;32:220-35.

40 Westhorp G, Manzano A. Realist Evaluation Interviewing-A 'Starter Set' of Questions. The RAMESES II Project. The National Institute for Health Research, 2017. Available: http://www.ramesesproject. org/media/RAMESES_II_Realist_interviewing_starter_questions.pdf [Accessed 14 Jan 2020].
41 QSR International Pty Ltd. NVivo, 2020. Available: https://www. qsrinternational.com/nvivo-qualitative-data-analysis-software/home [Accessed cited 2020 Jan 13]

42 Groot G, Waldron T, Carr T, et al. Development of a program theory for shared decision-making: a realist review protocol. Syst Rev 2017:6:114.

43 Wong G, Brennan N, Mattick K, et al. Interventions to improve antimicrobial prescribing of doctors in training: the IMPACT (IMProving Antimicrobial presCribing of doctors in Training) realist review. BMJ Open 2015;5:e009059.

44 Pawson R, Greenhalgh T, Harvey G, et al. Realist synthesis: an introduction. Manchester: University of Manchester, 2004. Available: emanticscholar.org/paper/Realist-synthesis-\%3A-an-introductionRay-Pawson-Greenhalgh-Harvey/435146e6e6617491ff1c4b32b76e 0a534c86d6c7 [Accessed cited 2019 Dec. 18] 